\title{
Subcutaneous emphysema and pneumothorax during percutaneous tracheostomy without any evidence of tracheal wall injury on repeated bronchoscopy
}

\author{
Mritunjay Kumar \\ Department of Anaesthesiology and Intensive Care, Govind Ballabh Pant Hospital, New Delhi, India
}

Percutaneous dilatational tracheostomy (PDT) is fast becoming the method of choice for securing an airway in chronic ventilated patients in intensive care units worldwide. It has been advocated as a safe and efficient bedside alternative for open surgical tracheostomy [1,2]. Most of the complications reported with different PDT techniques are mild and easy to overcome, but some major, life-threatening complications like pneumothorax, pneumomediastinum, subcutaneous emphysema, cardiovascular compromise have been reported as well.

Our patient was 50 years old female, weighing $84 \mathrm{~kg}$, with diagnosis of right hand marjolin's ulcer with emphysema and respiratory failure. Bedside PDT with ULTRAperc (Smith Medical, Hythe, Kent, UK) single stage dilator technique was planned, as she was on ventilator support for 11 days and was a difficult case to wean. After all aseptic precautions and local anesthetic infiltration, procedure was started under fiberoptic bronchoscope (FOB) guidance. Standard technique for PDT was followed; FOB was inserted through a special $\mathrm{T}$ connector up to the level of carina. After dilatation of the tracheal stoma, when the tracheostomy tube was inserted, end-tidal carbon dioxide trace was detected in the beginning but soon thereafter no visible chest rise was observed and on auscultation of the chest, no breath sounds were heard. The patient was immediately connected to the endotracheal tube, which was still lying in the oral cavity just below the vocal cords, still there was no visible chest rise and compliance of the chest was very poor. Patient also developed desaturation upto $\mathrm{SPO}_{2}$ of $88 \%$, bradycardia and hypotension. Cardiopulmonary resuscitation was started. Possibility of pneumothorax was thought upon and 16 gauge intravenous cannula with three way assembly leading to water seal was inserted in the left second intercostal space as decrease in breath sounds were more predominant on left side. There was a sudden gush of air. There was also air crepitus on palpation in the neck and chest wall on palpation. Intercostal drain was put on left side in fifth intercostal space along the midclavicular line. Then, tracheostomy was completed under bronchoscopic visualisation and $7 \mathrm{~mm}$ internal diameter tracheostomy tube was put into the stoma. Position was confirmed using, capnography, chest auscultation and fiberoptic bronchoscopy. The patient was also started on inotropic support and vitals improved gradually. No evidence of tracheal wall injury was found even on repeated bronchoscopy. The pneumothorax that occurred in this case could have been caused either by a false passage formation or by rupture of emphysematous bullae as a result of an increase in peak airway pressure and/ or air trapping. The dynamic hyperinflation of lungs and air trapped in the thorax and mediastinum could be implicated in decreasing the venous return and thereby causing hemodynamic compromise in the form of hypotension, bradycardia and near cardiac arrest.

Ambesh et al. [3] also reported pneumothorax after the Ciaglia Blue Rhino dilatation in an 80 year old patient, in which no injury to trachea was found on repeated bronchoscopy.

In study by Byhahn et al. [4], pneumothorax was detected radiologically in a patient after uncomplicated PDT. Chest tube

Corresponding author: Mritunjay Kumar, M.D., D.N.B., Department of Anaesthesiology and Intensive Care, Govind Ballabh Pant Hospital, New Delhi-110002, India. Tel: 919868023165, Fax: 919868023165, E-mail: dr.mritunjay@gmail.com

(c) This is an open-access article distributed under the terms of the Creative Commons Attribution Non-Commercial License (http:// creativecommons.org/licenses/by-nc/3.0/), which permits unrestricted non-commercial use, distribution, and reproduction in any medium, provided the original work is properly cited. 
insertion resulted in immediate clinical improvement, and the follow up was uneventful. Although bronchoscopic control during the tracheostomy procedure did not reveal any tracheal injury, a small tear in the posterior tracheal wall at the level of the puncture site was detected by an otorhinolarygolist seven days after PDT.

Fikkers et al. [5] in a retrospective analysis of 326 patients undergoing PDT in between 1997 and 2002 and described five cases of subcutaneous emphysema (1.5\%) and two cases of pneumothorax $(0.6 \%)$. In the simultaneous literature search, they found 41 cases of emphysema (1.4\%) and 25 cases of pneumothorax $(0.8 \%)$ in a total of 3,012 patients. They also analyzed the potential mechanisms for the development of emphysema and pneumothorax in human cadaver models. Various proposed mechanisms are damage to posterior tracheal wall, damage to anterior tracheal wall, false passage or paratracheal placement, dislocation of cannula, imperfect positioning of the fenestrated cannula and barotrauma.

Risk of puncturing the pleura is greater in chronic obstructive pulmonary disease and patients with emphysematous lung because of higher location of pleural dome and also when puncture site is lower.

Subcutaneous emphysema and pneumothorax is rare but disastrous complication of PDT. But, we should also keep in our mind other possible causes of subcutaneous emphysema and pneumothorax like rupture of emphysematous bullae and actively preclude it before blaming it on the PDT technique. Brocnchoscopic visualisation during the procedure to avoid damage to tracheal wall, selecting puncture site carefully, avoiding use of fenestrated tracheal tube at least during first week, careful selection of the patients and constant vigilance during the procedure is some of the measures that can be adopted to avoid these complications.

\section{References}

1. Friedman Y, Fildes J, Mizock B, Samuel J, Patel S, Appavu S, et al. Comparison of percutaneous and surgical tracheostomies. Chest 1996; 110: $480-5$.

2. Levin R, Trivikram L. Cost/benefit analysis of open tracheotomy, in the or and at the bedside, with percutaneous tracheotomy. Laryngoscope 2001; 111: 1169-73.

3. Ambesh SP, Pandey CK, Srivastava S, Agarwal A, Singh DK. Percutaneous tracheostomy with single dilatation technique: a prospective randomised comparison of Ciaglia blue rhino versus Griggs' guidewire dilating forceps. Anesth Analg 2002; 95: 1739-45.

4. Byhahn C, Wilke HJ, Halbig S, Lischke V, Westphal K. Percutaneous tracheostomy: ciaglia blue rhino versus the basic ciaglia technique of percutaneous dilational tracheostomy. Anesth Analg 2000; 91: 882-6.

5. Fikkers BG, van Veen JA, Kooloos JG, Pickkers P, van den Hoogen FJ, Hillen B, et al. Emphysema and pneumothorax after percutaneous tracheostomy: case reports and an anatomic study. Chest 2004; 125: 1805-14. 